KN Kartographische Nachrichten Journal of Cartography and Geographic Information 68. Jahrgang, Februar 2018, Heft 1 Herausgeber: Deutsche Gesellschaft für Kartographie e.V. (DGfK) - Gesellschaft für Kartographie und Geomatik, vertrete durch den Präsidenten Prof. Dr. Manfred Weisensee

Organ der Deutschen Gesellschaft für Kartographie e.V. (DGfK). der Schweizerischen Gesellschaft für Kartografie (SGK) und der Österreichischen Kartographischen Kommission (OKK) in der Österreichischen Gesellschaft für Geographie (ŌGG).

Schriftleitung

Hauptschriftleiter

Prof. Dr. rer. nat. habil. Mark Vetter (zuständig für Aufsătze, Berichte). Hochschule Karlsruhe - Technik und Wirtschaft,

Moltkestr. 30, 76133 Karlsruhe; redaktion@kartographische-

nachrichten.de, Fon: 0049(0) 7219252599

Weitere Schriftleiter

Prof. Dr. phil. Frank Dickmann (Zweiter Schriftleiter, zuständig für Nachrichten aus Hochschulen und Institutionen, Rezensionent. Ruhr-Universität Bochum, Geographisches Institut, Postfach, 44780 Bochum; frank.dickmann@rubde Fon: 0049(0) 234 32-23379; Fax: 0049(0) 234 32-14964 Dipl.-Ing. Andreas Gollenstede (zuständig für Geoinformation aktuell) Haareneschstr. 91, 26121 Oldenburg: kn@gollenstede.cor Fon: 0049(0) 441 7779-545; Fax: 0049(0) 441 7779-750 Dr.-Ing. Thomas Chudy (zustăndig für DGFK-, SKG- und ÖGGNachrichten). Martin-Luther-Universität Halle-Wittenberg, Institut für Agrar- und Ernährungswissenschaften, Karl-Freiherr-vonFritsch-Straße 4, 06120 Halle/Saale, Fon: 0049(0) 3455522448 ; Fax: 0049(0) 3455527109

Prof. Dr. Reinhard Zölitz (zuständig für Nachrichten aus Hochschulen und Institutionen), Institut für Geographie und Geologie, Kartographie und GIS, Friedrich-Ludwig-Jahn-Str. 16, D-17489 Greifswald, Fon 03834864523, Fax: 03834864501, E-Mail: zoelitz@uni-greifswald.de

Mitarbeit für die Schweiz: Prof. Dr. Lorenz Hurni. Institut für

Kartografie, ETH Hönggerberg, 8093 Zürich; hurni@karto.baug.ethz. ch. Fon:0041 16333033; Fax:0041 1631153

Mitarbeit für Österreich: Univ.-Prof. Dr. Wolfgang Kainz Institut für Geographie und Regionalforschung, Universität Wien, Universitätsstraße 7/5, 1010 Wien; wolfgang.kainz@univie.ac.at Fon: 0043 14277-8640; Fax: 0043 14277-9531

Ehrenschriftleiter

Prof. Dr. Jürgen Dodt t Witten

Dr. rer. nat. h. c. Rolf Harbeck, Bonn

Editorial Board

Prof. Dr. H. Asche, Potsdam; Prof. Dr. Manfred Buchroithner, Dresden; Prof. Dr. Dirk Burghardt, Dresden; Prof. Dr. D. Dransch, Potsdam; Prof. Dr. M. Ehlers, Osnabrück, Berlin; Prof. Dr. S. I. Fabrikant, Zürich, Schweiz; Ass.-Prof. Dr. S. Fuhrmann, Fairfax USA: Prof. Dr. G. Gartner, Wien, Österreich; Prof. Dr. D. Grünreich, Ronnenberg; Dr. Rolf Harbeck, Bonn; Prof. Dr. A. Hüttermann, Ronnenberg; Dr. Rolf Harbeck, Bonn; Prof. Dr. A. Huttermann,
Ludwigsburg; Prof. Dr. L. Hurni, Zürich; Prof. Dr. W. Kainz, Wien; Prof. Dr. P. Kammerer, München; Prof. Dr. W. G. Koch, Dresden; Prof. Dr. Menno-Jan Kroak, Twente, Netherlands; Prof. Dr. Jukka Krisp, Augsburg; Prof. Dr.-Ing. L Meng, Mūnchen: Prof. Dr. J. Schiewe, Hamburg; PD Dr.-Ing. J. Schoppmeyer, Bonn; Prof. Dr.J. Schweikart, Berlin; Prof. Dr-Ing. M. Sester, Hannover; Prof. Dr. J. Siemer, Regina Kanada, Prof. Dr. A. Zölitz, Greifswaid. Manuskripte richten Sie bitte an den zuständigen Schriftleiter. Hinweise zur Gestaltung des Manuskripts finden Sie unter www.kartographische-nachrichten.de > Autorenhinweise Reviewverfahren

Wissenschaftliche Beiträge werden im Rahmen eines Peer-ReviewVerfahrens begutachtet. Trotz sorgfältiger Redigierung übernehmen Herausgeber, Redaktion und Verlag keine Haftung für die inhalttiche Richtigkeit der Veröffentlichungen.

Die KN Kartographische Nachrichten ist in der internationalen Zitationsdatenbank Scopus gelistet.

Die DGfK ist Mitglied der

Internationalen Kartographischen

Vereinigung IKV/ICA.

Verlag: Kirschbaum Verlag GmbH

Siegfriedstraße 28, D-53179 Bonn

Postfach 210209, D-53157 Bonn

Fon: (0228) 95453-0, Fax: (0228) 95453-27

Internet: http://www.kirschbaum.de, E-Mail: info@kirschbaum.de

Anzeigenleiter: Michael Dietl Nerlagsleiter Fachzeitschriften prin Et digital), Fon: (0228) 95453-47. E-Mail: m.diet|@kirschbaum.de; Anzeigenpreisliste Nr. 53 vom 1.10.2017

Bezugspreise und ISSN: Inland/Ausland Jahresabonnement

inkl. E-Paper und elektronischem Archiv 74,- Euro zzgl.

Versand 9,80,- Euro (jeweils einschl. MwSt).

Einzelheft 18,- Euro zzgl. Versand

Die Zeitschrift erscheint alle zwei Monate. ISSN 0022-9164. Kündigungsfrist 6 Wochen zum Auslaufen des AbonnementZeitraumes.

Satz: EMS Eckert Medienservice, 53881 Euskirchen

Druck: johnen-druck GmbH \& Co. KG, Industriegebiet Bornwiese, 54470 Bernkastel-Kues

\section{Virtual Reality in der Kartographie}

\author{
Liebe Leserin, lieber Leser,
}

die Kartographie hat sich schon immer mit der Visualisierung räumlicher Strukturen befasst. Dies ist ihre Kernaufgabe, ihre Kernkompetenz. Dabei hat sie sich stets der Methoden bedient, die jeweils dem neuesten Stand der Technik entsprachen. So ist es nicht erstaunlich, dass gegenwärtig die technischen Entwicklungen im Zusammenhang mit der Virtual Reality (VR) für die Kartographie besonders spannend sind. Zu den Methoden, die für sie nutzbar sind, gehört das gesamte Spek-

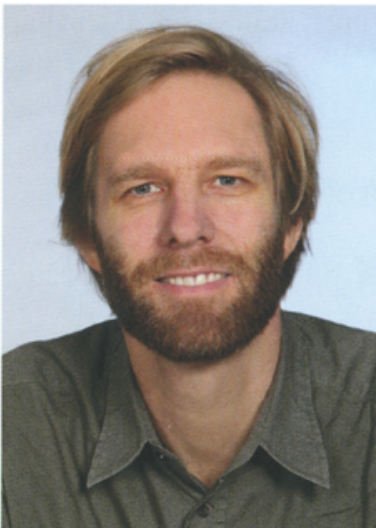

Mark Vetter trum der VR, somit auch die Varianten wie Mixed Reality, Augmented Reality oder Augmented Virtuality.

Schon jetzt stehen uns bezüglich der Hard- und Software faszinierende Werkzeuge zur Verfügung, die in den nächsten Jahren sicher technisch noch weiter an Reife gewinnen werden. Insbesondere an der Performanz von Soft- und Hardware, aber auch im Bereich der gerätespezifischen Auflösung der visuellen Präsentationsgeräte wird sich noch einiges tun. Vor allem die Controller, das sind die Interaktionskomponenten zwischen Mensch und Maschine, werden in Zukunft effizienter und intuitiver werden.

Softwarelösungen zur Modellierung und Visualisierung von VR-Welten gibt es schon seit langer Zeit. Der Vorläufer der Game Engine Unity 3D geht zurück ins Jahr 2005. Die erste Version der Unreal Engine ist sogar auf das Jahr 1998 datiert. Aber im Bereich der VR-Hardware hat es erst in den letzten Jahren einen Entwicklungsschub gegeben, sodass nun die „VR-Brillen“ für den Endverbraucher erschwinglich und nutzerfreundlich einzusetzen sind. Die ersten Geräte für den Verbraucher, der HTC Vive oder der Oculus Rift, wurden im März/April 2016 auf den Markt gebracht. Es dauert in der Regel einige Zeit, bis die Nutzerinnen und Nutzer, aber auch die Forschung darauf reagiert und bis alle Soft- und Hardwarekomponenten nahezu reibungslos miteinander arbeiten. Daher können wir wirklich noch von einer sehr jungen Entwicklung sprechen. Für die Forschung im Bereich der 3D-Kartographie ergibt sich folglich in den nächsten Jahren auf diesem jungen Fachgebiet ein großes Potenzial.

Lesen Sie also in dieser Ausgabe zur VR-Thematik und ihrem Potenzial für die 3D-Kartographie mit Open-Source-Produkten einen Artikel vom Autorenteam um Dennis Edler. Mit der Darstellung und Implementierung von Reisezeit-Kartogrammen haben sich Lina Wang et al. beschäftigt, und das Team um Jörg Hennersdorf löst auf, was Nichtwohnbaustrukturen sind und welchen Beitrag ATKIS-Daten für die Darstellung und Analyse dieser Strukturen leisten können.

Ich wünsche viel Freude beim Lesen des Heftes.

\section{Herzlichst,}

Ihr

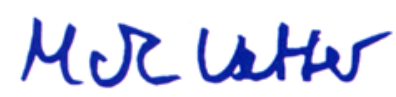

